\title{
Extramammärer Morbus Paget und Melanom: Zwei Fälle zweifacher Krebserkrankungen
}

\author{
Erika Lydrup $^{\mathrm{a}}$ Jais Oliver Berg ${ }^{\mathrm{a}} \quad$ Sofie Vetli Hjorth ${ }^{\mathrm{b}} \quad$ Lisbet Rosenkrantz Hølmich $^{\mathrm{a}}$ \\ Jørgen Lock-Andersen ${ }^{a}$ Annette Hougaard Chakera ${ }^{a}$
}

${ }^{a}$ Abteilung für Plastische Chirurgie, Herlev-Gentofte Hospital, Universitätsklinikum Kopenhagen, Herlev, Dänemark;

${ }^{b}$ Abteilung für Pathologie, Herlev-Gentofte Hospital, Universitätsklinikum Kopenhagen, Herlev, Dänemark

\author{
Schlüsselwörter \\ Extramammärer Morbus Paget · Melanom · zweifache maligne \\ Erkrankung
}

\section{Zusammenfassung}

Extramammärer Morbus Paget (EMP) ist eine seltene, intraepidermale neoplastische Erkrankung. Ein Zusammenhang zwischen EMP und zugrunde liegenden malignen Erkrankungen ist umfassend belegt. Jedoch liegen bisher erst wenige Berichte über Fälle von EMP in Kombination mit kutanen Melanomen vor. In diesem Fallbericht werden zwei solche Fälle von zweifacher maligner Er- krankung vorgestellt; ein Patient mit einem Kollisionstumor und eine Patientin mit zwei Neoplasien unterschiedlicher Lokalisation. Wir diskutieren die Pathogenese und Behandlung sowie die Bedeutung einer gründlichen klinischen und radiologischen Untersuchung und eine Literaturübersicht.

(c) 2020 Die Autoren. Herausgegeben von S. Karger AG, Basel.

\section{Einleitung}

Der extramammäre Morbus Paget (EMP) ist eine seltene, intraepidermale neoplastische Erkrankung, die durch juckende erythematöse Plaques in Hautarealen mit hoher Dichte apokriner Drüsen gekennzeichnet ist, z. B. Ohr, Vulva, Skrotum, Axilla, Inguinal- und Perianalregion $[1,2]$. Ein Zusammenhang des EMP mit zugrunde liegenden in-situ- oder invasiven Neoplasien ist umfassend belegt [1,2]. Über kutane maligne Melanome (MM) in Verbindung mit EMP liegen jedoch erst wenige Fallberichte vor, und zwar als Kollisionstumoren [3-5] oder gleichzeitig an unterschiedlichen Lokalisationen auftretend, mit primärem MM [6] oder rezidivierendem MM [7]. Im vorliegenden Fallbericht werden zwei solche Fälle von zweifacher maligner Erkrankung vorgestellt; ein Patient mit einem Kollisionstumor und eine Patientin mit zwei Neoplasien unterschiedlicher Lokalisation.

\section{Vorstellung der Fälle}

Der erste Fall war eine 69-jährige Frau, bei der 2 Jahre zuvor ein oberflächlich spreitendes MM am linken Arm diagnostiziert worden war (1,1 mm Tumordicke nach Breslow mit Mitoserate $\geq 1 / \mathrm{mm}^{2}$, jedoch ohne Ulzeration/Regression; klinisches Stadium IB). Die Behandlung bestand in einer weiträumigen lokalen Exzision mit $2 \mathrm{~cm}$ Sicherheitsabstand und einer Sentinel-Lymphknoten-Biopsie der linken Axilla, die keine Metastasierung ergab. Die postoperative Nachbeobachtung war ereignislos, bis nach 2 Jahren die Patientin ein juckendes Exanthem am Perineum bemerkte. Er wurde als großflächige ekzematöse Läsion von 3-4 cm auf der linken Seite der Perianalhaut beschrieben, die etwa ein Drittel der Zirkumferenz des Anus bedeckte und keine tiefere Infiltration zeigte. Eine Biopsie ergab EMP. Eine gynäkologische Untersuchung und transrektaler Ultraschall blieben ohne Be- 


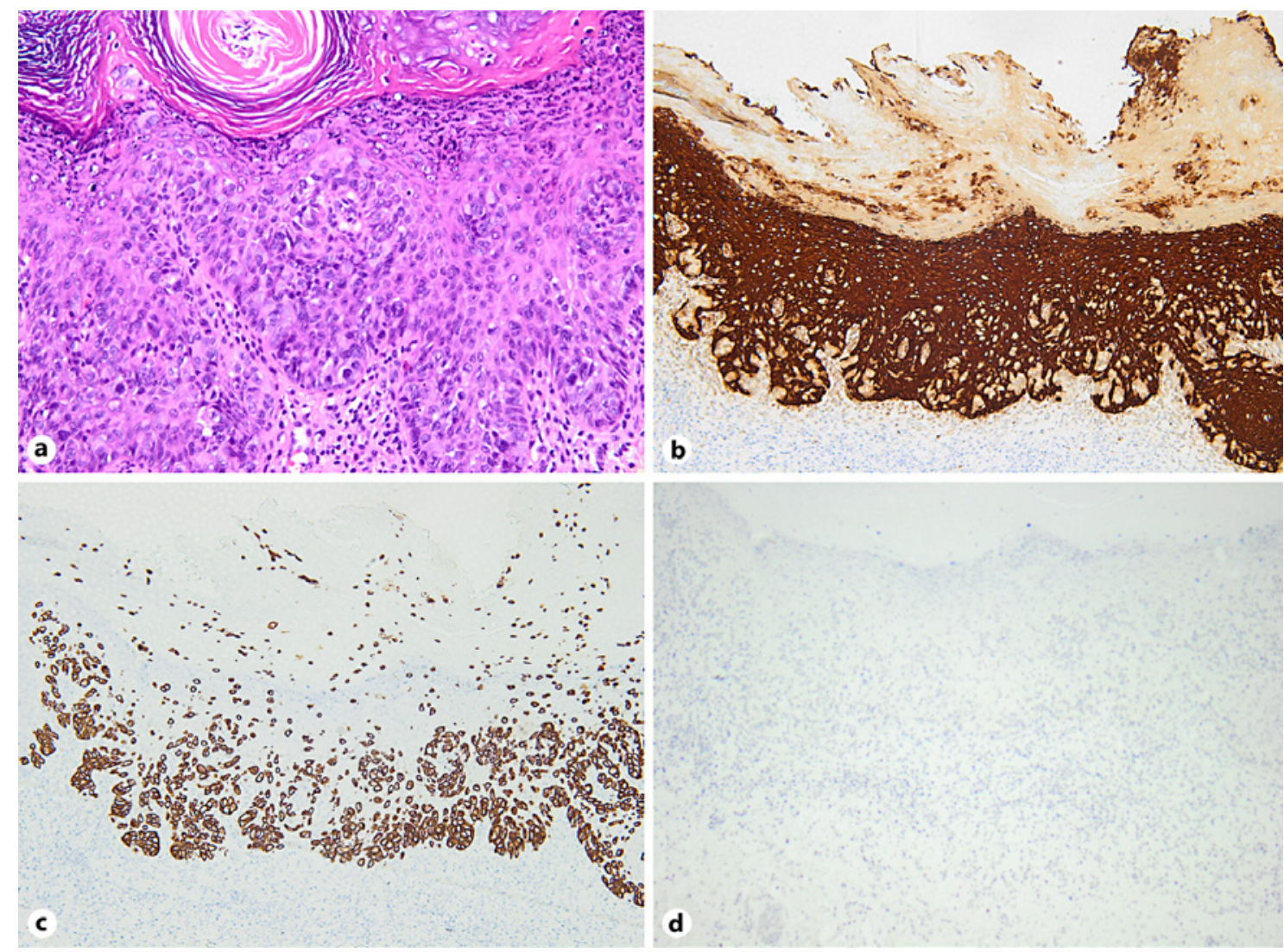

Abb. 1. a-d. Histologische Darstellung der perianalen Haut mit Paget-Zellen in der Epidermis. a. Neoplastische Zellen mit abundantem Zytoplasma und vergrößerten vesikulären Zellkernen mit prominenten Nucleoli liegen sowohl in Form konfluierender Nester als auch als einzelne Zellen in der Epidermis verteilt vor. Hämatoxylin-Eosin, $\times 20$. Die Tumorzellen waren negativ für CK5 (b), zeigten jedoch Färbung für CK7 (c). d. Die Tumorzellen exprimieren Ber-EP4, weshalb extramammärer Morbus Paget diagnostiziert werden kann. Immunohistologische Färbung; Vergrößerung: ×10. Alle Zellen waren negativ für Sox10 (immunohistologische Färbung).

fund. Die Läsion wurde chirurgisch mit $1 \mathrm{~cm}$ Saum entfernt und der Defekt mit einem Nahlappen rekonstruiert. Im PET-CT zeigte sich ein einzelner FDG-positiver Lymphknoten in der linken Axilla, ohne weitere verdächtige Herde. Eine ultraschallgesteuerte Biopsie ergab Metastasen des MM. Eine linksseitige radikale Dissektion der axillären Lymphknoten wurde durchgeführt und ergab Metastasen in einem von 27 Lymphknoten, ohne perinodalen Befall. Seitdem wird die Patientin in einem Programm für Patienten mit hohem Rezidivrisiko nachbeobachtet; das Programm umfasst klinische Kontrolluntersuchungen alle 3 Monate in der Abteilung für plastische Chirurgie sowie routinemäßige PET-CT-Scans nach 6, 12, 24 und 36 Monaten oder bei entsprechender Indikation auch früher. Zusätzlich dazu erfolgen alle 6 Monate für 2 Jahre eine klinische Kontrolluntersuchung und Anoskopie in der Abteilung für Allgemeinchirurgie. Eine Koloskopie 3 Monate nach der Operation war ohne Befund, und nach 15-monatiger Nachbeobachtung war die Patientin rezidivfrei (Abb. 1).

Der zweite Patient war ein 86-jähriger Mann mit Behinderung durch bleibende Folgen eines Schlaganfalls, bei dem ein Prostatakarzinom und multiple Basalzellkarzinome in der Vorgeschichte vorlagen. Der Patient stellte sich mit einer umfangreichen Läsion hinter und unter dem linken Ohr vor. Zwei Jahre zuvor hatte eine Biopsie eine aktinische Keratose (von nicht klassifiziertem Typ) ergeben, und die Läsion war mit Imiquimod-Creme behandelt worden. Angesichts des Wachstums der Läsion wurde eine erneute Biopsie durchgeführt, die ein MM ergab - ungewiss, ob Primärtumor oder Metastase. Das PET-CT war ohne Anzeichen maligner Erkrankung an anderer Stelle. Der Patient wurde einer ausgedehnten lokalen Exzision der Läsion mit $1 \mathrm{~cm}$ Sicherheitsabstand unterzogen, und die histopathologische Untersuchung ergab ein nicht klassifiziertes MM mit abundanten Spindelzellen (Lentigo maligna wurde in Betracht gezogen, jedoch erfüllte die Läsion nicht die Kriterien) und EMP in demselben Areal sowie unvollständige Resektionsränder. Bei der Nachresektion wurde festgestellt, dass in allen Proben aus dem Tumorareal EMP vorlag. Der Patient war nicht in der Verfassung für eine größere Operation unter Allgemeinanästhesie, eine Strahlentherapie lehnte er ab. Aufgrund eines Lokalrezidivs sowohl des MM als auch des EMP 9 Monate später willigte der Patient in eine palliative Strahlentherapie ein. Die Surveillance besteht aus klinischen Kontrolluntersuchungen (Abb. 2, 3). 


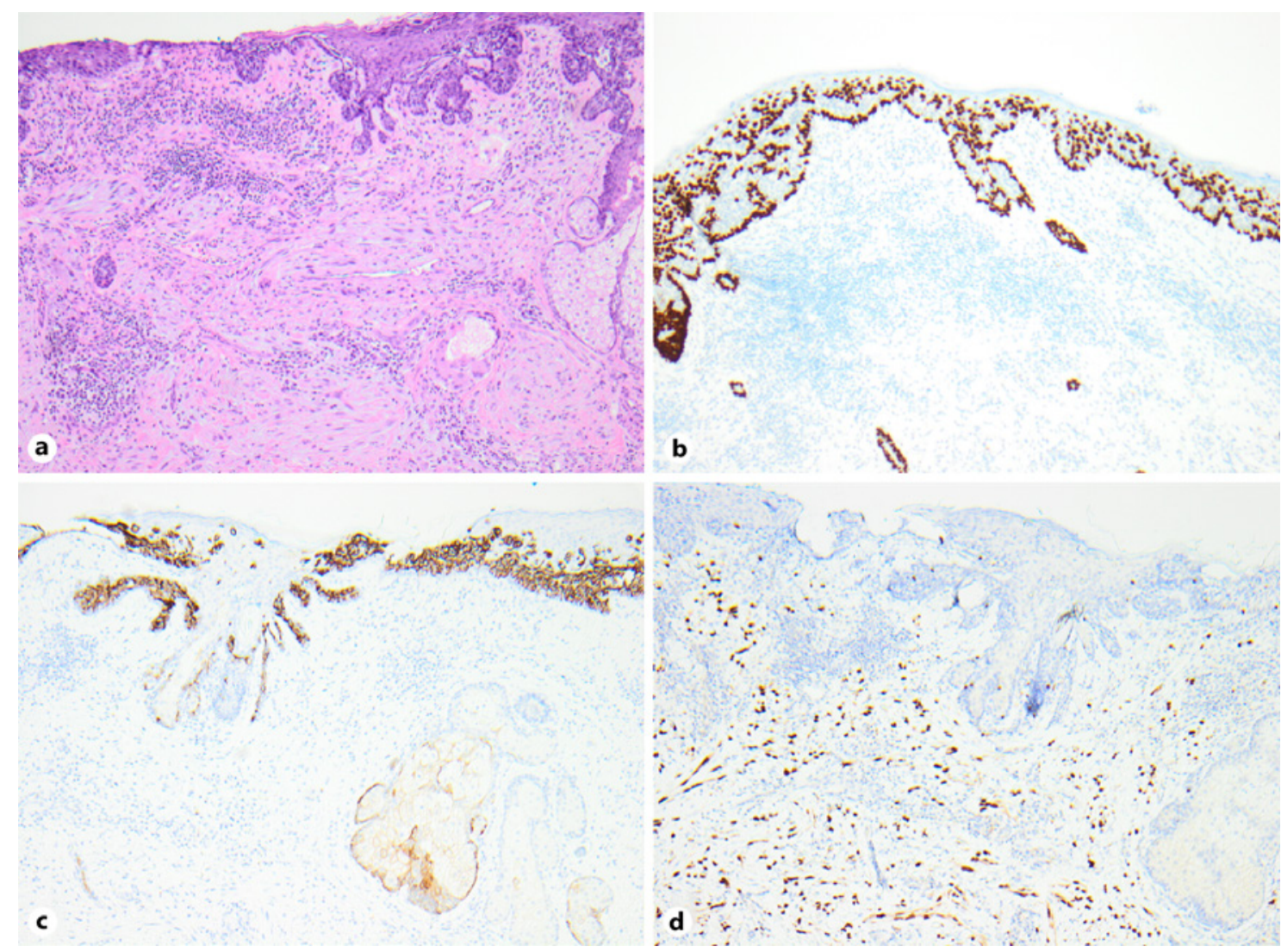

Abb. 2. Histologische Darstellung der postaurikulären Haut neben dem Melanom. Paget-Zellen liegen in der Epidermis und im adnexalen Epithel der Dermis vor. a. Pagetoide Verteilung von Paget-Zellen in der Epidermis. Hämatoxylin-Eosin, $\times 20$. b. Die Tumorzellen waren negativ für P40, gesundes Epithel hingegen positiv. c. Positive Färbung für CK7. Immunhistologische Färbungen; Vergrößerung: ×10. d. Sox10-Färbung eines invasiven Melanoms. Immunhistologische Färbung; Vergrößerung: ×10. Immunhistologische Färbungen: CK7: Cytokeratin 7; P40: Protein 40; Ber-EP4: Epitheliales Zelladhäsionsmolekül; Sox10: Sox10-Protein.

\section{Diskussion}

James Paget beschrieb 1874 erstmals einen Zusammenhang zwischen Morbus Paget (MP) der Mamma und zugrundeliegendem Mammakarzinom, und 1889 erfolgte durch H.R. Crocker die Erstbeschreibung eines EMP des Skrotums und Penis [1]. Bei Frauen ist die Vulva die häufigste Lokalisation eines EMP, gefolgt von der perianalen Region $[1,2]$. Die Erkrankung tritt typischerweise im Alter von 50-80 Jahren auf, am häufigsten bei Frauen kaukasischer Abstammung [2, 8].

Etwa 70\% der Patienten stellen sich mit Pruritis vor, der das häufigste Symptom ist [2]. Aufgrund seines unspezifischen Erscheinungsbilds wird EMP häufig als entzündliche oder infektiöse Hauterkrankung fehldiagnostiziert (z.B. als Ekzem, seborrhoische Dermatitis oder Psoriasis), wie in unserem zweiten Fall beschrieben, und es ist daher nicht ungewöhnlich, dass die Läsion sich bereits in einem fortgeschritten Stadium befindet, wenn die korrekte Diagnose gestellt und eine adäquate Behandlung eingeleitet wird [1].

Aktuell gilt die Theorie, dass der EMP in den meisten Fällen als primäre intraepidermale Neoplasie (primärer EMP) oder, seltener, infolge der Ausbreitung einer zugrunde liegenden internen

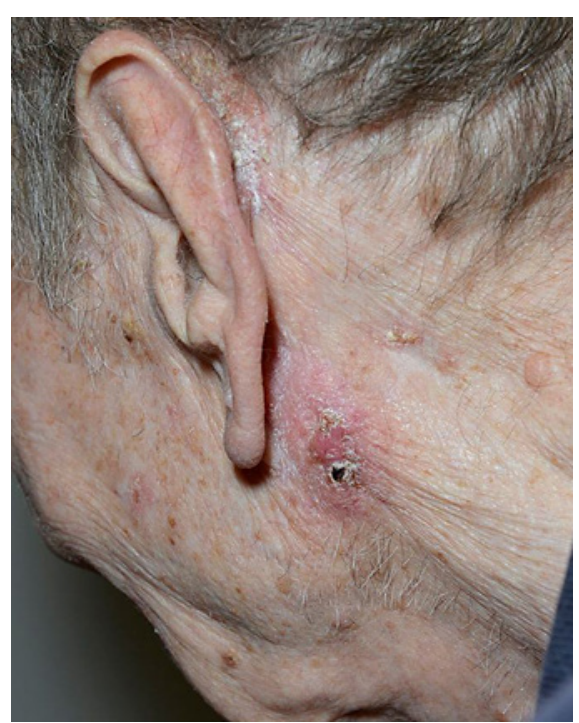

Abb. 3. Klinische Fotografie der Läsion hinter dem linken Ohr. Die Biopsie ergab MM und EMP in diesem Areal. 
malignen Erkrankung (sekundärer EMP) entsteht. Der primäre EMP kann zu einem dermal invasiven Adenokarzinom fortschreiten und, wenn er unbehandelt bleibt, in lokale Lymphknoten oder entfernte Lokalisationen metastasieren. Der sekundäre EMP hingegen entsteht durch epidermotrope Ausbreitung maligner Zellen aus einer zugrunde liegenden Neoplasie $[1,2]$.

Einige Stimmen bringen EMP mit einer generellen Neigung zu Neoplasien, insbesondere Adenokarzinomen, in Verbindung. EMP der Vulva ist mit Neoplasien in Endometrium, Endozervix, Vagina, Vulva, Urethra und Blase in Zusammenhang gebracht worden, während EMP der äußeren männlichen Genitalien mit Neoplasien in Blase, Urethra und Prostata in Verbindung gebracht werden kann. Man nimmt an, dass durchschnittlich 25\% aller Fälle von EMP mit anderen neoplastischen Erkrankungen assoziiert sind, wobei die Häufigkeit je nach Lokalisation der Erkrankung variiert [1]. Bei vulvärem EMP sind 11-20\% der Fälle mit einem zugrunde liegenden viszeralen Malignom assoziiert [ 9 , 10]. Perianaler EMP ist seltener als vulvärer EMP, ist jedoch in 14-45\% der Fälle eng mit Adenokarzinomen des Anus und des Kolorektums assoziiert $[1,11,12]$. In den meisten Fällen kann man durch eine sorgfältige morphologische Evaluierung und mithilfe eines Panels von immunhistologischen Markern zur korrekten Diagnose gelangen. Die häufigsten Differenzialdiagnosen sind Melanom und atypische Plattenepithelerkrankungen.

Die Rate der Lokalrezidivierung ist hoch, was darauf zurückzuführen sein könnte, dass einige klinische Merkmale des EMP unregelmäßige Ränder, Multizentrizität und die Tendenz zur Beteiligung gesund erscheinender Haut - die radikale Exzision erschweren [2]. Eine Studie [10], die auf 100 Fällen basiert, ergab eine Rezidivrate von 34\% nach einer medianen Dauer von 3 Jahren. Eine andere Studie [13] mit 30 Fällen ermittelte eine Rezidivrate von 44\%, wobei Patienten mit invasiver Erkrankung eine höhere Rate an Lokalrezidiven aufwiesen als solche mit in-situ-Erkrankung.

Die Standardbehandlung des EMP ist die weiträumige lokale Exzision mit einem Sicherheitsabstand von mindestens $1 \mathrm{~cm}$ [1]. Um die Erfolgsaussicht auf saubere Ränder zu erhöhen, sollte bei der pathologischen Untersuchung eine Immunhistochemie durchgeführt werden, und gegebenenfalls sollten zusätzliche Schnitte der Gewebeprobe vorgenommen werden. Auch Gefrierschnitte können durchgeführt werden.

Aufgrund der hohen Häufigkeit assoziierter maligner Erkrankungen sollte die EMP-Diagnostik mit gründlichen Untersuchungen einhergehen, die z.B. eine Koloskopie, Sigmoidoskopie, Röntgenthorax oder PET-CT umfassen können. Nachbeobachtung ist bei Patienten mit diagnostiziertem EMP unerlässlich und muss auch langfristig fortgeführt werden. Sie sollte sowohl das lokale Rezidivrisiko als auch assoziierte interne Malignome berücksichtigen [2].
Unseres Wissens liegen bisher erst 5 Berichte über Fälle von sowohl EMP als auch MM vor [3-7]. EMP und rezidivierendes MM kommen extrem selten vor; wir haben lediglich einen Fallbericht gefunden [7]. Die Literatur über den möglichen Zusammenhang zwischen EMP und MM ist spärlich, und es liegt bisher keine Evidenz zu seiner Existenz vor. Tsuji et al. [6] diskutierten dies und postulierten, dass unterschiedliche genetische Mechanismen, die EMP und Melanom zugrunde liegen, einen allgemeinen Mangel an Assoziation erklären könnten.

Im vorliegenden Fallbericht stellen wir 2 verschiedene Fälle von MM und EMP vor. Im ersten Fall liegen ein rezidivierendes MM und EMP vor; hier zeigte sich, dass der PET-CT-Scan ein wertvolles Untersuchungsinstrument ist, wenn bei einem Patienten EMP diagnostiziert wird. Beim zweiten Fall mit einem Kollisionstumor von MM und EMP sind zwei Aspekte von besonderem Interesse: Erstens wird EMP häufig fehldiagnostiziert, was die Diagnosestellung hinauszögert. Zweitens macht die Multifokalität der Läsion eine äußerst sorgfältige pathologische Untersuchung des Präparats erforderlich. Es ist möglich, dass unsere Fälle nur zwei Zufälle darstellen. Wir möchten jedoch die Wahrscheinlichkeit eines Zusammenhangs zwischen EMP und MM hervorheben und eine Ganzkörper-Hautuntersuchung als Teil der klinischen Untersuchung bei allen Patienten empfehlen, bei denen EMP diagnostiziert wird, da die Erkrankung mit MM assoziiert sein könnte. PET-CT-Scans sind nur begrenzt geeignet, kleinere Tumoren der Haut zu erkennen.

\section{Ethikerklärung}

Die Autoren haben keine ethische Konflikte offenzulegen. Diese Forschungsarbeit wurde ethisch in Übereinstimmung mit der Deklaration von Helsinki des Weltärztebundes durchgeführt. Die Patienten haben ihr schriftliches Einverständnis auch zur Verwendung der Fotografien erteilt.

\section{Disclosure Statement}

Die Autoren haben keine Interessenkonflikte offenzulegen.

\section{Finanzierungsquellen}

Für diese Arbeit wurde keine finanzielle Unterstützung geleistet.

\section{Beiträge der einzelnen Autoren}

Alle Autoren leisteten wesentliche Beiträge zu allen folgenden Aspekten: (1) Konzeption und Aufbau der Arbeit, (2) Texterstellung oder kritische Überarbeitung in wichtigen inhaltlichen Aspekten, (3) finale Freigabe der zu veröffentlichenden Version und (4) Einverständnis, für alle Aspekte der Arbeit verantwortlich zu sein und sicherzustellen, dass Fragen zur Richtigkeit oder Integrität jeglicher Teile der Arbeit angemessen untersucht und gelöst werden.

\section{Literatur}

Die Literatur ist unter www.karger.com/doi/10.1159/000517397 abrufbar. 